MATHEMATICS OF COMPUTATION

Volume 71, Number 240, Pages 1597-1607

S 0025-5718(01)01399-0

Article electronically published on December 5, 2001

\title{
SOME NUMERICAL COMPUTATIONS CONCERNING SPINOR ZETA FUNCTIONS IN GENUS 2 AT THE CENTRAL POINT
}

\author{
WINFRIED KOHNEN AND MICHAEL KUSS
}

\begin{abstract}
We numerically compute the central critical values of odd quadratic character twists with respect to some small discriminants $D$ of spinor zeta functions attached to Seigel-Hecke eigenforms $F$ of genus 2 in the first few cases where $F$ does not belong to the Maass space. As a result, in the cases considered we can numerically confirm a conjecture of Böcherer, according to which these central critical values should be proportional to the squares of certain finite sums of Fourier coefficients of $F$.
\end{abstract}

\section{INTRODUCTION}

In [3], Böcherer made an interesting conjecture concerning central critical values of odd quadratic character twists of spinor zeta functions attached to cuspidal Siegel-Hecke eigenforms of genus 2.

More precisely, let $F$ be a nonzero cuspidal Hecke eigenform of even integral weight $k$ w.r.t. the Siegel modular group $\Gamma_{2}:=\operatorname{Sp}_{2}(\mathbb{Z})$ and denote by $Z_{F}(s)$ $(\operatorname{Re}(s) \gg 0)$ its spinor zeta function. Recall [2] that $Z_{F}(s)$ completed with appropriate $\Gamma$-factors has a meromorphic continuation to $\mathbb{C}$ and is invariant under $s \mapsto 2 k-2-s$. Let $Z_{F}\left(s, \chi_{D}\right)(\operatorname{Re}(s) \gg 0)$ be the twist of $Z_{F}(s)$ by the quadratic character $\chi_{D}=\left(\frac{D}{.}\right)$, where $D<0$ is a fundamental discriminant. Assume that $Z_{F}\left(s, \chi_{D}\right)$ enjoys similar analytic properties as $Z_{F}(s)$. Then according to [3], there should exist a constant $C_{F}>0$, depending only on $F$, such that

$$
Z_{F}\left(k-1, \chi_{D}\right)=C_{F}|D|^{1-k}\left(\sum_{\{T>0 \mid \operatorname{discr} T=D\} / \Gamma_{1}} \frac{a(T)}{\varepsilon(T)}\right)^{2},
$$

where $a(T)$ ( $T$ a positive definite half-integral $(2,2)$-matrix) is the $T$-th Fourier coefficient of $F, \varepsilon(T):=\#\left\{U \in \Gamma_{1} \mid T[U]=T\right\}$ (with $\Gamma_{1}:=\mathrm{SL}_{2}(\mathbb{Z}), T[U]=U^{t} T U$ ) is the order of the unit group of $T$ and the summation in (1) extends over all $T$ with discriminant equal to $D$, modulo the action $T \mapsto T[U]$ by $\Gamma_{1}$.

In [3, Böcherer proved his conjecture in the case where $F$ is the Maass lift of a Hecke eigenform $f$ of weight $2 k-2$ w.r.t. $\Gamma_{1}$. The proof combines four inputs: i) the fact that $Z_{F}(s)=\zeta(s-k+1) \zeta(s-k+2) L(f, s)$, where $L(f, s)$ is the Hecke $L$-function of $f$ [5]; ii) Waldspurger's theorem [13] on the relation between central critical values of quadratic twists of $L(f, s)$ and squares of Fourier coefficients of modular forms of half-integral weight; iii) the explicit description of the Maass

Received by the editor October 20, 1999 and, in revised form, January 3, 2001.

2000 Mathematics Subject Classification. Primary 11F46.

(C)2001 American Mathematical Society 
lift on the level of Fourier coefficients [2]; and finally iv) Dirichlet's classical class number formula.

Later on, Böcherer and Schulze-Pillot 4 proved an identity similar to (1) in the case of levels, where now $F$ is the Yoshida lift of an elliptic cusp form.

Also in [3], a formula like (10) in the case where $F$ is a Siegel- or KlingenEisenstein series was shown to be true.

The proof in all the above cases makes essential use of the fact that the spinor zeta function in question is a product of "known" $L$-series.

To the best of our knowledge, nothing regarding Böcherer's conjecture seems to be known in the case where $F$ is a "true" Siegel modular form, i.e., is not a lift of an automorphic form on $\mathrm{GL}_{2}$ (and so $Z_{F}(s)$ is not expected to split).

In the present paper, we would like to present some numerical data supporting the conjecture for small values of $D$ in the first few "nontrivial" cases when $F$ is of weight 20,22, 24 resp. 26 and is not a Maass lift. It turns out that for those $F$ and for $D=-3,-4,-7,-8$ identity (1) numerically is true at least up to 5 digits with some constant $C_{F}>0$ independent of $D$ (Thm., $\S 4$; numerical data are given in $\S 5)$.

The first ingredient in the computation is a certain series representation (found by the first author many years ago) for central critical values of spinor zeta functions supposing "good" analytic properties of $Z_{F}\left(s, \chi_{D}\right)$ as required in the conjecture. We were kindly informed by D. Goldfeld that this series representation can also be derived from the more general work of Lavrik [10] when appropriately specialized. The formula for computing $Z_{F}\left(k-1, \chi_{D}\right)$ is given in $\S 2$.

Note that the holomorphic continuation of $Z_{F}\left(s, \chi_{D}\right)$ was proved in [6], [7] (using some round-about via Rankin-Dirichlet series) under the assumption that the first Fourier-Jacobi coefficient of $F$ is nonzero. The latter condition is satisfied at least for all $F$ with $k \leqslant 32$ according to Skoruppa [12]. The functional equation, however, was proved only very recently in [9].

The second main ingredient, which is entirely due to the second author, is the computation of the eigenvalues $\lambda_{F}(p)(p$ a prime $<1000)$ and $\lambda_{F}\left(p^{2}\right)(p$ a prime $<71$ ) under the usual Hecke operators $T_{p}$ resp. $T_{p^{2}}$ of the $F$ in question, following the method of Skoruppa [12] and an appropriate $\mathrm{C}++$ computer program. This is presented in $\S 3$.

In $\S 4$, the results of $\S \S 2$ and 3 are combined to calculate $Z_{F}\left(k-1, \chi_{D}\right)$ for the $F$ and $D$ in question with "good" accuracy. For an estimation of the error term we use the bounds for the eigenvalues of $F$ implied by the Ramanujan-Petersson conjecture, for the latter cf. [14].

We finally remark that we have also numerically re-checked (1) using the identity given in $\S 2$ in case $F$ is of weight 20, resp. 22, and is in the Maass space. We have not included the details here.

\section{A SERIES REPRESENTATION FOR CENTRAL VALUES OF SPINOR ZETA FUNCTIONS}

Let $k \in 2 \mathbb{N}$ and write $S_{k}\left(\Gamma_{2}\right)$ for the space of Siegel cusp forms of weight $k$ w.r.t. $\Gamma_{2}$. If $F \in S_{k}\left(\Gamma_{2}\right)$ is a nonzero Hecke eigenform, we let

$$
Z_{F}(s)=\prod_{p \text { prime }} Z_{F, p}\left(p^{-s}\right)^{-1} \quad(\operatorname{Re}(s) \gg 0)
$$


be the spinor zeta function of $F$, where

$$
\begin{aligned}
Z_{F, p}(X)= & 1-\lambda_{F}(p) X+\left(\lambda_{F}(p)^{2}-\lambda_{F}\left(p^{2}\right)-p^{2 k-4}\right) X^{2} \\
& -\lambda_{F}(p) p^{2 k-3} X^{3}+p^{4 k-6} X^{4}
\end{aligned}
$$

is the local spinor polynomial at $p$ and $\lambda_{F}(p)$ resp. $\lambda_{F}\left(p^{2}\right)$ are the eigenvalues of $F$ under the usual Hecke operator $T_{p}$ resp. $T_{p^{2}}$.

According to Andrianov [2] the function

$$
Z_{F}^{\star}(s)=(2 \pi)^{-2 s} \Gamma(s) \Gamma(s-k+2) Z_{F}(s)
$$

has a meromorphic continuation to $\mathbb{C}$ and is invariant under $s \mapsto 2 k-2-s$. It is holomorphic everywhere if $F$ is not contained in the Maass space (which is equivalent to saying $Z_{F}(s)$ is not of the form $Z_{F}(s)=\zeta(s-k+1) \zeta(s-k+2)$ $\times L(f, s)$, where $f$ is a normalized cuspidal Hecke eigenform of weight $2 k-2$ w.r.t. $\Gamma_{1}$, and $L(f, s)$ is its associated Hecke $L$-function [5]).

If $D<0$ is a fundamental discriminant, we define the twist of $Z_{F}(s)$ by $\chi_{D}$ as

$$
Z_{F}\left(s, \chi_{D}\right):=\prod_{p \text { prime }} Z_{F, p}\left(\chi_{D}(p) p^{-s}\right)^{-1} \quad(\operatorname{Re}(s) \gg 0) .
$$

We denote the $n$-th coefficient of the Dirichlet series $Z_{F}\left(s, \chi_{D}\right)$ by $\lambda_{F, D}(n)$.

We put

$$
Z_{F}^{\star}\left(s, \chi_{D}\right):=\left(\frac{2 \pi}{|D|}\right)^{-2 s} \Gamma(s) \Gamma(s-k+2) Z_{F}\left(s, \chi_{D}\right) \quad(\operatorname{Re}(s) \gg 0) .
$$

If $F$ is in the Maass space, then by well-known properties of twists of $\zeta(s)$ and $L(f, s), Z_{F}^{\star}\left(s, \chi_{D}\right)$ extends to an entire function, is of rapid decay for $\operatorname{Im}(s) \rightarrow \infty$ and is invariant under $s \mapsto 2 k-2-s$. It is very natural to expect that the same holds for general $F$ (cf. [3]). In fact, if $F$ is not in the Maass space and the first Fourier-Jacobi coefficient of $F$ is nonzero, this was proved in [6], 7], 9] (using the fact $\left\|\phi_{1}\right\|^{2} Z_{F}(s)=D_{F}(s)$, where $\phi_{1}$ is the first Fourier-Jacobi coefficient of $F$ and $D_{F}(s)$ is a Rankin type Dirichlet series formed out of the Fourier-Jacobi coefficients of $F$ introduced in [8]).

Let $F \in S_{k}\left(\Gamma_{2}\right)$ be a Hecke eigenform such that $Z_{F}\left(s, \chi_{D}\right)$ has the above analytic properties. Using the integral transform

$$
\frac{1}{2 \pi i} \int_{c-i \infty}^{c+i \infty} \Gamma(s) \Gamma(s-k+2) y^{-s} \mathrm{~d} s=2 y^{-\frac{k}{2}+1} K_{k-2}(2 \sqrt{y}) \quad(y>0, c>k-2),
$$

where $K_{k-2}(y)$ denotes the modified Bessel function of order $k-2$, we have for $y>0$ and $c \gg 0$

$$
\begin{aligned}
& \frac{1}{2 \pi i} \int_{c-i \infty}^{c+i \infty} Z_{F}^{\star}\left(s, \chi_{D}\right) y^{-s} \mathrm{~d} s \\
& \quad=\frac{1}{2 \pi i} \int_{c-i \infty}^{c+i \infty}\left(\frac{2 \pi}{|D|}\right)^{-2 s} \Gamma(s) \Gamma(s-k+2) \sum_{n=1}^{\infty} \lambda_{F, D}(n) n^{-s} y^{-s} \mathrm{~d} s \\
& \quad=\sum_{n=1}^{\infty} \lambda_{F, D}(n) \frac{1}{2 \pi i} \int_{c-i \infty}^{c+i \infty} \Gamma(s) \Gamma(s-k+2)\left(\frac{4 \pi^{2} n y}{D^{2}}\right)^{-s} \mathrm{~d} s \\
& \quad=y^{-\frac{k}{2}+1} f_{F, D}(y),
\end{aligned}
$$


where

$$
f_{F, D}(y)=2\left(\frac{4 \pi^{2}}{D^{2}}\right)^{-\frac{k}{2}+1} \sum_{n=1}^{\infty} \lambda_{F, D}(n) n^{-\frac{k}{2}+1} K_{k-2}\left(\frac{4 \pi \sqrt{n y}}{|D|}\right) .
$$

Since $Z_{F}^{\star}\left(s, \chi_{D}\right)$ is holomorphic and of rapid decay for $\operatorname{Im} s \rightarrow \infty$, we may shift the path of integration in (4) to the line $c=k-1$. We replace $y$ by $\frac{1}{y}$ and apply the functional equation of $Z_{F}^{\star}\left(s, \chi_{D}\right)$ to obtain

$$
\begin{aligned}
y^{\frac{k}{2}-1} & f_{F, D}\left(\frac{1}{y}\right) \\
& =\frac{1}{2 \pi i} \int_{k-1-i \infty}^{k-1+i \infty} Z_{F}^{\star}\left(s, \chi_{D}\right) y^{s} \mathrm{~d} s=\frac{1}{2 \pi i} \int_{k-1-i \infty}^{k-1+i \infty} Z_{F}^{\star}\left(2 k-2-s, \chi_{D}\right) y^{s} \mathrm{~d} s \\
& =\frac{1}{2 \pi i} \int_{k-1-i \infty}^{k-1+i \infty} Z_{F}^{\star}\left(s, \chi_{D}\right) y^{2 k-2-s} \mathrm{~d} s=y^{\frac{3}{2} k-1} f_{F, D}(y),
\end{aligned}
$$

i.e., the function $f_{F, D}(y)$ satisfies the functional equation $f_{F, D}\left(\frac{1}{y}\right)=y^{k} f_{F, D}(y)$.

Using the usual splitting trick and the formula

$$
2 \int_{0}^{\infty} K_{k-2}(2 \sqrt{y}) y^{s-\frac{k}{2}} \mathrm{~d} y=\Gamma(s) \Gamma(s-k+2) \quad(\operatorname{Re}(s)>k-2),
$$

we conclude for $\operatorname{Re}(s) \gg 0$ that

$$
\begin{aligned}
Z_{F}^{\star}\left(s, \chi_{D}\right) & =2\left(\frac{2 \pi}{|D|}\right)^{-2 s} \sum_{n=1}^{\infty} \lambda_{F, D}(n) n^{-s} \int_{0}^{\infty} K_{k-2}(2 \sqrt{y}) y^{s-\frac{k}{2}} \mathrm{~d} y \\
& =2\left(\frac{2 \pi}{|D|}\right)^{-2 s} \sum_{n=1}^{\infty} \lambda_{F, D}(n) n^{-s}\left(\frac{4 \pi^{2} n}{D^{2}}\right)^{s-\frac{k}{2}+1} \int_{0}^{\infty} K_{k-2}\left(\frac{4 \pi \sqrt{n y}}{|D|}\right) y^{s-\frac{k}{2}} \mathrm{~d} y \\
& =\int_{0}^{\infty} f_{F, D}(y) y^{s-\frac{k}{2}} \mathrm{~d} y=\int_{1}^{\infty} f_{F, D}(y)\left(y^{\frac{3}{2} k-2-s}+y^{s-\frac{k}{2}}\right) \mathrm{d} y
\end{aligned}
$$

As $f_{F, D}(y)$ is of exponential decay for $y \rightarrow \infty$, the right hand side of (5) has a holomorphic continuation to the whole complex plane, and (5) is valid for all $s \in \mathbb{C}$.

Setting $s=k-1$ in (5), we get the formulas

$$
\begin{aligned}
Z_{F}^{\star}\left(k-1, \chi_{D}\right) & =4\left(\frac{4 \pi^{2}}{D^{2}}\right)^{-\frac{k}{2}+1} \int_{1}^{\infty} \sum_{n=1}^{\infty} \lambda_{F, D}(n) n^{-\frac{k}{2}+1} K_{k-2}\left(\frac{4 \pi \sqrt{n y}}{|D|}\right) y^{\frac{k}{2}-1} \mathrm{~d} y \\
& =4\left(\frac{4 \pi^{2}}{D^{2}}\right)^{-\frac{k}{2}+1} \sum_{n=1}^{\infty} \int_{1}^{\infty} \lambda_{F, D}(n) n^{-\frac{k}{2}+1} K_{k-2}\left(\frac{4 \pi \sqrt{n y}}{|D|}\right) y^{\frac{k}{2}-1} \mathrm{~d} y \\
& =4\left(\frac{4 \pi^{2}}{D^{2}}\right)^{-\frac{k}{2}+1} \sum_{n=1}^{\infty} \lambda_{F, D}(n) n^{-k+1} \int_{n}^{\infty} K_{k-2}\left(\frac{4 \pi \sqrt{y}}{D \mid}\right) y^{\frac{k}{2}-1} \mathrm{~d} y
\end{aligned}
$$

Hence

$$
Z_{F}\left(k-1, \chi_{D}\right)=\frac{4(2 \pi)^{k}}{|D|^{k}(k-2) !} \sum_{n=1}^{\infty} \lambda_{F, D}(n) n^{-k+1} \int_{n}^{\infty} K_{k-2}\left(\frac{4 \pi \sqrt{y}}{|D|}\right) y^{\frac{k}{2}-1} \mathrm{~d} y
$$

where the exponential decay of $K_{k-2}(y)$ for $y \rightarrow \infty$ justifies the interchange of summation and integration in ([6). 


\section{NumERICAL COMPUTATIONS}

Let $M_{k}\left(\Gamma_{1}\right)$ be the space of elliptic modular forms of weight $k$ w.r.t. $\Gamma_{1}$ and $S_{k}\left(\Gamma_{1}\right)$ be the subspace of cusp forms in $M_{k}\left(\Gamma_{1}\right)$. For $\tau \in \mathbb{C}, \operatorname{Im}(\tau)>0$, write $q=\exp (2 \pi i \tau)$, and let

$$
\Delta=q \prod_{n=1}^{\infty}\left(1-q^{n}\right)^{24}
$$

be the Ramanujan $\Delta$-function in $S_{12}\left(\Gamma_{1}\right)$ and

$$
\begin{gathered}
E_{2 k}=1-\frac{4 k}{B_{2 k}} \sum_{n=1}^{\infty} \sigma_{2 k-1}(n) q^{n} \quad\left(k \in \mathbb{Z}, k \geqslant 2, \sigma_{2 k-1}(n)=\sum_{d \mid n} d^{2 k-1},\right. \\
\left.B_{2 k}=2 k \text { th Bernoulli number }\right)
\end{gathered}
$$

be the normalized Eisenstein series in $M_{2 k}\left(\Gamma_{1}\right)$.

If $J_{k, 1}^{\text {cusp }}$ denotes the space of Jacobi cusp forms on $\Gamma_{1}$ of index 1 and weight $k$, the Maass space [11] is the image of the Hecke equivariant embedding $V: J_{k, 1}^{\text {cusp }} \hookrightarrow$ $S_{k}\left(\Gamma_{2}\right)$ defined by

$$
\begin{aligned}
\phi= & \sum_{\substack{D, r \in \mathbb{Z}, D<0 \\
D \equiv r^{2} \bmod 4}} C_{\phi}(D) q^{\left(r^{2}-D\right) / 4} \zeta^{r} \\
\longmapsto & \sum_{\substack{n, r, m \in \mathbb{Z} \\
r^{2}-4 m n<0, n, m>0}} a(n, r, m) q^{n} \zeta^{r} q^{\prime m},
\end{aligned}
$$

where

$$
a(n, r, m):=\sum_{d \mid(n, r, m)} d^{k-1} C_{\phi}\left(\frac{r^{2}-4 m n}{d^{2}}\right)
$$

and

$$
\zeta=\exp (2 \pi i z)(z \in \mathbb{C}), \quad q^{\prime}=\exp \left(2 \pi i \tau^{\prime}\right)\left(\tau^{\prime} \in \mathbb{C}, \operatorname{Im}\left(\tau^{\prime}\right)>0\right)
$$

By $\phi_{10}$ resp. $\phi_{12}$ we denote the Jacobi cusp forms in the one-dimensional spaces $J_{10,1}^{\text {cusp }}$, resp. $J_{12,1}^{\text {cusp }}$, normalized to $C(-3)=1$.

The first cuspidal Hecke eigenforms for genus 2 that do not belong to the Maass space appear in weight $20,22,24$, resp. 26 , and are denoted $\Upsilon_{20}, \ldots, \Upsilon_{26 b}$ in [12]. In [12, Skoruppa gives explicit formulas for them (involving the forms $V(\phi)$, where $\phi$ are appropriate Jacobi forms) and calculates some of their Fourier coefficients. Note that there is a misprint in the formula for $\Upsilon_{22}$; the corrected formula is

$$
\begin{aligned}
\Upsilon_{22}= & -2^{5} \cdot 3 \cdot 5 \cdot 7 \cdot 1423 \cdot V\left(\phi_{10}\right) V\left(\phi_{12}\right) \\
& +V\left(-\frac{5}{2 \cdot 3} \phi_{12} E_{10}+\frac{11}{2 \cdot 3} \phi_{10} E_{6}^{2}+2^{4} \cdot 3 \cdot 61 \cdot \phi_{10} \Delta\right) .
\end{aligned}
$$


To compute the coefficients of the relevant Jacobi forms $\phi$, we proceed slightly differently from [12] and try to avoid multiplication of Jacobi modular forms with elliptic modular forms. More precisely, the operator $\mathcal{D}_{2 \nu}$ is defined by

$$
\mathcal{D}_{2 \nu} \phi:=\sum_{n=0}^{\infty}\left(\sum_{r} p_{2 \nu}^{(k-1)}(r, n m) c(n, r)\right) q^{n} \quad(\nu \in \mathbb{Z}, \nu \geqslant 0)
$$

where $\phi=\sum_{n, r} c(n, r) q^{n} \zeta^{r} \in J_{k, m}^{\text {cusp }}$ and

$$
\frac{(k-\nu-2) !}{(2 \nu) !(k-2) !} p_{2 \nu}^{(k-1)}(r, n)=\text { coefficient of } t^{2 \nu} \text { in }\left(1-r t+n t^{2}\right)^{-k+1}
$$

$\operatorname{maps} J_{k, 1}^{\text {cusp }}$ to $S_{k+2 \nu}\left(\Gamma_{1}\right)[5]$.

We consider the system of equations $\left\{\mathcal{D}_{2 \nu}(f)=g_{f, \nu}\right\}$ where $f$ is one of the Jacobi forms

$$
\begin{aligned}
& \phi_{10}, \phi_{10} E_{4}, \phi_{10} E_{6}, \phi_{10} E_{10}, \phi_{10} E_{14}, \phi_{10} E_{16}, \phi_{10} \Delta, \phi_{10} E_{6}^{2}, \phi_{10} E_{8}^{2}, \phi_{10} \Delta E_{4}, \\
& \phi_{12}, \phi_{12} E_{8}, \phi_{12} E_{10}, \phi_{12} E_{6}^{2}, \phi_{12} \Delta, \phi_{12} E_{14},
\end{aligned}
$$

$\nu \in\{0,2,4\}$ and $g_{f, \nu}$ is the corresponding elliptic modular form which is determined by its first coefficients, e.g., we have

$$
\begin{array}{lll}
\mathcal{D}_{0}\left(\phi_{10}\right)=0, & \mathcal{D}_{2}\left(\phi_{10}\right)=20 \Delta, & \mathcal{D}_{4}\left(\phi_{10}\right)=0, \\
\mathcal{D}_{0}\left(\phi_{12}\right)=12 \Delta, & \mathcal{D}_{2}\left(\phi_{12}\right)=0, & \mathcal{D}_{4}\left(\phi_{12}\right)=196 \Delta E_{4} .
\end{array}
$$

We solve the system recursively for the Fourier coefficients of the Jacobi forms. (To start the recursion the first Fourier coefficients of $\phi_{10}, \phi_{12}$ are taken from [5].) This method needs only $O\left(|D|^{\frac{3}{2}}\right)$ operations to calculate a complete table of Fourier coefficients up to a "large" discriminant. Hence it is less "expensive" than the usual multiplication of Jacobi forms and elliptic modular forms $\left(O\left(|D|^{2}\right)\right)$.

Proceeding in this way and using a $\mathrm{C}++$ computer program, we computed the Fourier coefficients $C(D)$ of the Jacobi forms in question for $|D| \leqslant 3000000$. Then we are able to compute any Fourier coefficient $a(n, r, m)$ of $\Upsilon_{20}, \ldots, \Upsilon_{26 b}$ with discriminant $4 m n-r^{2} \leqslant 3000000$.

In 12] Skoruppa calculates the eigenvalues $\lambda_{F}(p), \lambda_{F}\left(p^{2}\right)(p$ prime) of a Hecke eigenform

$$
F=\sum_{\substack{r, n, m \in \mathbb{Z}, r^{2}-4 m n<0, n, m>0}} a(n, r, m) q^{n} \zeta^{r} q^{\prime m} \in S_{k}\left(\Gamma_{2}\right)
$$

by means of the formulas

$$
\lambda_{F}(p) a(1,1,1)=a(p, p, p)+p^{k-2}\left(1+\left(\frac{p}{3}\right)\right) a(1,1,1)
$$

and

$$
\begin{aligned}
\lambda_{F}\left(p^{2}\right) a(1,1,1) & \\
= & {\left[\lambda_{F}(p)^{2}-\lambda_{F}(p) p^{k-2}\left(1+\left(\frac{p}{3}\right)\right)-p^{2 k-3}+p^{2 k-4}\left(\left(\frac{p}{3}\right)+\left(\frac{p}{3}\right)^{2}\right)\right] a(1,1,1) } \\
& -p^{k-2} a\left(1, p, p^{2}\right)-p^{k-2} \sum_{\substack{\nu \bmod p, 1+\nu+\nu^{2} \neq 0 \bmod p}} a\left(1+\nu+\nu^{2}, p(1+2 \nu), p^{2}\right),
\end{aligned}
$$

which are based on Andrianov's results in [2]. 
Using another $\mathrm{C}++$ computer program, we computed the eigenvalues $\lambda_{F}(p)$ for $p<1000$ prime and $\lambda_{F}\left(p^{2}\right)$ for $p<71$ prime of $F=\Upsilon_{20}, \ldots, \Upsilon_{26 b}$ from the above formulas.

\section{Summing UP}

By (7) we have

$$
Z_{F}\left(k-1, \chi_{D}\right)=\sum_{n=1}^{\infty} \lambda_{F, D}(n) g_{D}(n)
$$

where

$$
g_{D}(n)=\frac{4(2 \pi)^{k}}{|D|^{k}(k-2) !} n^{-k+1} \int_{n}^{\infty} K_{k-2}\left(\frac{4 \pi \sqrt{y}}{|D|}\right) y^{\frac{k}{2}-1} \mathrm{~d} y .
$$

Now $g_{D}(n)$ is of exponential decay for $n \rightarrow \infty$ and $\lambda_{F, D}(n)$ is of polynomial growth. Thus for a numerical approximation of $Z_{F}\left(k-1, \chi_{D}\right)$ it is important to calculate as many terms as possible in the sum for small $n$ (say $n \leqslant N$ for some $N$ - we will later choose $N=4000)$, while for large $n(n>N)$ the total sum of all terms with $n>N$ is rather small. Hence we approximate $Z_{F}\left(k-1, \chi_{D}\right)$ by

$$
\mathcal{Z}_{F, D}(k-1)=\sum_{\substack{1 \leqslant n \leqslant N \\ n \text { has no prime } \\ \text { divisor }>1000}} \lambda_{F, D}(n) g_{D}(n)
$$

where the values of $\lambda_{F, D}(n)$ can be calculated from the Euler product of $Z_{F, D}\left(s, \chi_{D}\right)$ for $n<71^{2}$.

Suppose there are positive constants $C_{1}, C_{2}, \alpha, \beta$ such that the estimates $\left|\lambda_{F}(p)\right|$ $\leqslant C_{1} \cdot p^{\alpha}$ ( $p$ prime) and $\left|\lambda_{F}(n)\right| \leqslant C_{2} \cdot n^{\beta}(n>N)$ hold. Then the error term

$$
\varepsilon(F, D)=Z_{F, D}(k-1)-\mathcal{Z}_{F, D}(k-1)
$$

can be estimated by

$$
\begin{aligned}
|\varepsilon(F, D)| \leqslant & \sum_{\substack{p>1000 \\
p \text { prime } \\
1 \leqslant \nu \leqslant N / p}}\left|\lambda_{F, D}(\nu p)\right| g_{D}(\nu p) \\
& +\sum_{n>N}\left|\lambda_{F, D}(\nu n)\right| \int_{n}^{\infty} K_{k-2}\left(\frac{4 \pi \sqrt{y}}{|D|}\right) y^{\frac{k}{2}-1} \mathrm{~d} y .
\end{aligned}
$$

Suppose now that $N<1007^{2}$. Then clearly for the first sum $\sum_{1}$ in the above equation we have the estimate

$$
\begin{aligned}
\sum_{1} & =\sum_{\substack{p>1000 \\
p \text { prime } \\
1 \leqslant \nu \leqslant N / p}}\left|\lambda_{F, D}(\nu) \lambda_{F}(p)\right| g_{D}(\nu p) \\
& \leqslant C_{1} \sum_{\substack{p>1000 \\
p \text { prime } \\
1 \leqslant \nu \leqslant N / p}}\left|\lambda_{F, D}(\nu)\right| p^{\alpha} g_{D}(\nu p)
\end{aligned}
$$


The second sum $\sum_{2}$ in (9) satisfies

$$
\begin{aligned}
\frac{|D|^{k}(k-2) !}{4(2 \pi)^{k}} \sum_{2} & =\sum_{n>N}\left|\lambda_{F, D}(n)\right| n^{-k+1} \int_{n}^{\infty} K_{k-2}\left(\frac{4 \pi \sqrt{y}}{|D|}\right) y^{\frac{k}{2}-1} \mathrm{~d} y \\
& \leqslant C_{2} \sum_{n>N} n^{\beta-k+1} \int_{n}^{\infty} K_{k-2}\left(\frac{4 \pi \sqrt{y}}{|D|}\right) y^{\frac{k}{2}-1} \mathrm{~d} y \\
& \leqslant C_{2} \sum_{n>N} \int_{n}^{\infty} K_{k-2}\left(\frac{4 \pi \sqrt{y}}{|D|}\right) y^{\beta-\frac{k}{2}} \mathrm{~d} y \\
& \leqslant C_{2} \sum_{n>N} \sum_{m \geqslant n} \int_{m}^{m+1} K_{k-2}\left(\frac{4 \pi \sqrt{y}}{|D|}\right) y^{\beta-\frac{k}{2}} \mathrm{~d} y \\
& \leqslant C_{2} \sum_{m>N}(m-N) \int_{m}^{m+1} K_{k-2}\left(\frac{4 \pi \sqrt{y}}{|D|}\right) y^{\beta-\frac{k}{2}} \mathrm{~d} y \\
& \leqslant C_{2} \int_{N+1}^{\infty} K_{k-2}\left(\frac{4 \pi \sqrt{y}}{|D|}\right) y^{\beta-\frac{k}{2}}(y-N) \mathrm{d} y .
\end{aligned}
$$

For the estimation of the dominating term $\sum_{1}$ in $\varepsilon(F, D)$ we use the result of Weissauer [14] that any eigenform $F \in S_{k}\left(\Gamma_{2}\right)$ which does not belong to the Maass space fulfills the Ramanujan-Petersson conjecture (i.e., all complex roots of $Z_{F, p}$ have absolute value $p^{\frac{3}{2}-k}$ ). Thus we have to choose $C_{1}=4, \alpha=k-\frac{3}{2}$ to obtain the best estimate for $\sum_{1}$ possible by our methods.

The contribution of $\sum_{2}$ to $\varepsilon(F, D)$ is absorbed by $\sum_{1}$ if $N$ is large enough, so we do not have to use the optimal estimate for $\lambda_{F}(n)$. One obtains a very crude (but simple and for our purpose sufficient) estimate for $\lambda_{F}(n)$ from the RamanujanPetersson conjecture if one uses $\sigma_{0}(n) \leqslant n$, namely

$$
\left|\lambda_{F}(n)\right| \leqslant \sum_{d \mid n} \sigma_{0}(d) \sigma_{0}\left(\frac{n}{d}\right) n^{k-\frac{3}{2}} \leqslant \sum_{d \mid n} n^{k-\frac{1}{2}}=\sigma_{0}(n) n^{k-\frac{1}{2}} \leqslant n^{k+\frac{1}{2}}
$$

Thus we set $C_{2}=1$ and $\beta=k+\frac{1}{2}$.

We choose $N=4000$ (then $\sum_{2}$ is dominated by $\sum_{1}$ for the $D$ in question) and calculate the numerical approximations of $Z_{F}\left(k-1, \chi_{D}\right)$ and the corresponding error terms using Mathematica. From (1) we computed the constants $C_{F}$ for $F=\Upsilon_{20}$, $\ldots, \Upsilon_{26 b}$ and $D=-3,-4,-7,-8$. The numerical results have been checked using Maple.

We obtain

Theorem. For $F=\Upsilon_{20}, \ldots, \Upsilon_{26 b}$ there are constants $C_{F}$ such that equation (1) (i.e., Böcherer's conjecture) holds for $D=-3,-, 4-7,-8$ numerically up to 5 digits. 


\section{NumericAl DATA}

TABLE 1. Approximate constants $C_{F}$ for $F=\Upsilon_{20}, \Upsilon_{22}$

\begin{tabular}{|r|l|l|}
\hline$D$ & $C_{\Upsilon_{20}}$ & $C_{\Upsilon_{22}}$ \\
\hline-3 & $2.0672152028688 \cdot 10^{11} \pm 0.5 \cdot 10^{-2}$ & $1.3056685268290 \cdot 10^{12} \pm 0.5 \cdot 10^{-1}$ \\
-4 & $2.0672152028688 \cdot 10^{11} \pm 0.5 \cdot 10^{-2}$ & $1.3056685268290 \cdot 10^{12} \pm 0.5 \cdot 10^{-1}$ \\
-7 & $2.0672152029206 \cdot 10^{11} \pm 2.9 \cdot 10^{1}$ & $1.3056685268295 \cdot 10^{12} \pm 1.1 \cdot 10^{1}$ \\
-8 & $2.0672152028644 \cdot 10^{11} \pm 3.1 \cdot 10^{1}$ & $1.3056685179067 \cdot 10^{12} \pm 1.1 \cdot 10^{6}$ \\
\hline
\end{tabular}

TABLE 2. Approximate constants $C_{F}$ for $F=\Upsilon_{24 a}, \Upsilon_{24 b}$

\begin{tabular}{|r|l|l|}
\hline$D$ & $C_{\Upsilon_{24 a}}$ & $C_{\Upsilon_{24 b}}$ \\
\hline-3 & $1.0953372445194 \cdot 10^{13} \pm 0.5 \cdot 10^{0}$ & $6.1388052839296 \cdot 10^{11} \pm 0.5 \cdot 10^{-2}$ \\
-4 & $1.0953372445194 \cdot 10^{13} \pm 0.5 \cdot 10^{0}$ & $6.1388052839296 \cdot 10^{11} \pm 0.5 \cdot 10^{-2}$ \\
-7 & $1.0953372445111 \cdot 10^{13} \pm 8.7 \cdot 10^{2}$ & $6.1388052891963 \cdot 10^{11} \pm 9.3 \cdot 10^{3}$ \\
-8 & $1.0953372445386 \cdot 10^{13} \pm 2.6 \cdot 10^{4}$ & $6.1388034612038 \cdot 10^{11} \pm 1.3 \cdot 10^{6}$ \\
\hline
\end{tabular}

TABLE 3. Approximate constants $C_{F}$ for $F=\Upsilon_{26 a}, \Upsilon_{26 b}$

\begin{tabular}{|r|l|l|}
\hline$D$ & $C_{\Upsilon_{26 a}}$ & $C_{\Upsilon_{26 b}}$ \\
\hline-3 & $9.6155285745891 \cdot 10^{13} \pm 0.5 \cdot 10^{0}$ & $6.2328839505417 \cdot 10^{12} \pm 0.5 \cdot 10^{-1}$ \\
-4 & $9.6155285745891 \cdot 10^{13} \pm 0.5 \cdot 10^{0}$ & $6.2328839505417 \cdot 10^{12} \pm 0.5 \cdot 10^{-1}$ \\
-7 & $9.6155285746522 \cdot 10^{13} \pm 1.1 \cdot 10^{4}$ & $6.2328839505729 \cdot 10^{12} \pm 2.3 \cdot 10^{2}$ \\
-8 & $9.6155285333968 \cdot 10^{13} \pm 8.2 \cdot 10^{6}$ & $6.2328839821394 \cdot 10^{12} \pm 1.6 \cdot 10^{5}$ \\
\hline
\end{tabular}

TABLE 4. The first Fourier coefficients of $\Upsilon_{20}, \ldots, \Upsilon_{26 b}$

\begin{tabular}{|r|l|r|r|r|r|r|r|}
\hline$D$ & $n, r, m$ & $\Upsilon_{20}$ & $\Upsilon_{22}$ & $\Upsilon_{24 a}$ & $\Upsilon_{24 b}$ & $\Upsilon_{26 a}$ & $\Upsilon_{26 b}$ \\
\hline-3 & $1,1,1$ & 1 & 1 & 1 & 3 & 1 & 3 \\
-4 & $1,0,1$ & 4 & -12 & -16 & 76 & -8 & 124 \\
-7 & $1,1,2$ & 56 & 1344 & 4408 & -616 & -7456 & 51632 \\
-8 & $1,0,2$ & 2616 & 216 & 44256 & -2904 & 15216 & -109752 \\
-11 & $1,1,3$ & -55077 & 409779 & -1147701 & 2122593 & -1180509 & 7299177 \\
-12 & $1,0,3$ & 408832 & 468448 & -378272 & 11995968 & 3505408 & -39833376 \\
-12 & $2,2,2$ & -840960 & -2215680 & -795324 & 18309504 & 9218340 & 495227520 \\
\hline
\end{tabular}


TABLE 5. The first eigenvalues of $\Upsilon_{20}$

\begin{tabular}{|c|c|}
\hline$n$ & $\lambda(n)$ \\
\hline 2 & -840960 \\
\hline 3 & 346935960 \\
\hline 5 & -5232247240500 \\
\hline 7 & 2617414076964400 \\
\hline 11 & 1427823701421564744 \\
\hline 13 & -83773835478688698980 \\
\hline 17 & 14156088476175218899620 \\
\hline 19 & 146957560176221097673720 \\
\hline 23 & -7159245922546757692913520 \\
\hline 29 & 1055528218470800414110149180 \\
\hline 31 & 4031470549468367403585068224 \\
\hline 37 & -154882657977740251483442365940 \\
\hline 41 & 1126683124934949617518831346964 \\
\hline 43 & 74572686686194644813168430600 \\
\hline 47 & -13773335595379978013820602730720 \\
\hline 53 & 29292488702536161643591933657260 \\
\hline 59 & 521943213201995351655113144025960 \\
\hline 61 & 896978197899858751399574623768444 \\
\hline 67 & -2921787486641381474027809454434280 \\
\hline $2^{2}$ & 248256200704 \\
\hline $3^{2}$ & -452051040393665991 \\
\hline $5^{2}$ & -94655785156653029446859375 \\
\hline $7^{2}$ & -5501629950184780949434983315951 \\
\hline $11^{2}$ & -126258221861417704499584077355164268151 \\
\hline $13^{2}$ & 2528254555352510520887488261241887242369 \\
\hline $17^{2}$ & 262144933510286336089464293262250165947750889 \\
\hline $19^{2}$ & -283417759450334375466210009895464677379295086759 \\
\hline $23^{2}$ & 127862428522278879932688110084314434400497569566129 \\
\hline $29^{2}$ & 408550299154535330723926336201059419422405306949883361 \\
\hline $31^{2}$ & -9417686481892622568784061821415683057728289096885473471 \\
\hline $37^{2}$ & 4270657975661931417960508434757260969748219593839247065169 \\
\hline $41^{2}$ & 129620395091878626890240343719327738119688391311944613269369 \\
\hline $43^{2}$ & -2 118391905744174698890014439813915105652042393393982400772151 \\
\hline $47^{2}$ & 10717867956150312430187083192735560357439349298395760667696609 \\
\hline $53^{2}$ & -6359983052359692969866068986893310598482880773029488944413754191 \\
\hline $59^{2}$ & 159291906542794821742879348124552646753906149121778952350318431721 \\
\hline $61^{2}$ & -653805853261332407170328486766159640869797840457778124369821593951 \\
\hline $67^{2}$ & 25254882862606589034647035623760404781292970925413106240956567868089 \\
\hline
\end{tabular}




\section{REFERENCES}

[1] M. Abramowitz, I. Stegun: Pocketbook of mathematical functions. Verlag Harri Deutsch, (1984). MR 85j:00005b

[2] A. Andrianov: Euler products corresponding to Siegel modular forms of genus 2. Russ. Math. Surveys 29, No.3, 45-116 (1974). MR 55:5540

[3] S. Böcherer: Bemerkungen über die Dirichletreihen von Koecher und Maass. Math. Gottingensis, Schriftenr. d. Sonderforschungsbereichs Geom. Anal. 68, (1986).

[4] S. Böcherer, R. Schulze-Pillot: The Dirichlet series of Koecher and Maass and modular forms of weight 3/2. Math. Z. 209, No.2, 273-287 (1992). MR 93b:11053

[5] M. Eichler, D. Zagier: The theory of Jacobi forms. Progress in Mathematics, Vol. 55. BostonBasel-Stuttgart: Birkhäuser (1985). MR 86j:11043

[6] W. Kohnen: On character twists of certain Dirichlet series. Mem. Fac. Sci. Kyushu Univ., vol. 47, 103-117 (1993). MR 94c:11044

[7] W. Kohnen, J. Sengupta, A. Krieg: Characteristic twists of a Dirichlet series for Siegel cusp forms. Manuscripta Math. 87, 489-499 (1995). MR 96f:11071

[8] W. Kohnen, N.-P. Skoruppa A certain Dirichlet series attached to Siegel modular forms of degree two. Invent. Math. 95, 541-558 (1989). MR 90b:11050

[9] M. Kuß: Die getwistete Spinor Zeta Funktion und die Böcherer Vermutung. Dissertation. (2000)

[10] A.F. Lavrik: Functional equations of Dirichlet functions. Soviet Math. Dokl. 7, 1471-1473 (1966). MR 34:4464

[11] H. Maass: Ueber eine Spezialschar von Modulformen zweiten Grades. I, II, III Invent. Math. 52, 95-104 (1979), Invent. Math. 53, 249-253, 255-265 (1979). MR 80f:10031 MR 81a:11037. MR 81a:11038

[12] N.P. Skoruppa: Computations of Siegel modular forms of genus two. Math. Comput. 58, 381-398 (1992). MR 92e:11041

[13] J.L. Waldspurger: Sur les coefficients de Fourier des formes modulaires de poids demi-entier. J. Math. Pures Appl. (9) 60, 375-484 (1981). MR 83h:10061

[14] R. Weissauer: The Ramanujan conjecture for genus two Siegel modular forms (an application of the trace formula). Preprint, Mannheim (1993)

Universität Heidelberg, Mathematisches Institut, Im Neuenheimer Feld 288, D-69120 Heidelberg, Germany

E-mail address: winfried@mathi.uni-heidelberg.de

Universität Heidelberg, Mathematisches Institut, Im Neuenheimer Feld 288, D-69120 Heidelberg, Germany

E-mail address: michael.kuss@urz.uni-heidelberg.de 